\title{
Trypanosomatidae de importancia en salud pública en animales silvestres y sinantrópicos en un área rural del municipio Tovar del estado Mérida, Venezuela
}

\author{
Héctor de Lima ${ }^{1}$, José Carrero 2, Armando Rodríguez 1, Zoraya de Guglielmo 1, \\ Noris Rodríguez ${ }^{1}$ \\ 1 Instituto de Biomedicina, Universidad Central de Venezuela, Caracas, Venezuela. \\ 2 Servicio de Dermatología Regional de Tovar, Módulo Las Acacias, Tovar, Mérida, Venezuela.
}

Introducción. La enfermedad de Chagas y la leishmaniasis son patologías de gran importancia en salud pública debido a su alta frecuencia y amplia distribución en América Latina. El estudio de los animales reservorios es vital para una comprensión global de estas enfermedades.

Objetivo. En el presente trabajo nos propusimos identificar parásitos de la familia Trypanosomatidae en animales silvestres con la finalidad de establecer posibles relaciones debidas a la coinfección.

Materiales y métodos. En El Carrizal, un área rural del estado Mérida, Venezuela, entre julio de 1998 y febrero de 2000 se realizaron capturas sistemáticas de animales silvestres con la finalidad de poner en evidencia la presencia de parásitos pertenecientes a la familia Trypanosomatidae. Las capturas fueron realizadas con trampas caseras tipo Tomahawk, colocadas 15 noches por mes durante el periodo del estudio. A los animales capturados se les practicó punción cardiaca bajo anestesia para extracción de sangre con la cual se realizaron los siguientes estudios: examen en fresco, extendido para coloración con Giemsa y cultivo en agar-sangre. Se realizaron algunos xenodiagnósticos. Los aislamientos obtenidos por medio de cultivo se identificaron por medio de análisis de restricción e hibridación con sondas específicas.

Resultados. Se capturaron y estudiaron 215 animales pertenecientes a tres especies: Rattus spp. (135), Sigmodon hispidus (73) y Didelphis marsupialis (7). Igualmente, se identificaron tres especies de Trypanosomatidae: Leishmania $(V)$ guyanensis, Trypanosoma cruzi y Trypanosoma lewisi. Mientras T. cruzi fue identificado en D. marsupialis (4/7), S. hispidus (1/73) y Rattus spp. (1/135), L.(V) guyanensis y T. lewisi sólo se identificaron en Rattus spp. (1/ 135 y $12 / 135$, respectivamente).

Conclusión. El estudio de la coexistencia de estas diferentes especies de flagelados genéticamente relacionados nos parece de importancia por las interacciones inmunológicas que se pueden establecer en los animales reservorios y las posibles implicaciones que esto puede tener en el huésped susceptible. Finalmente, las identificaciones de $L$. $(V)$ guyanensis y $T$. cruzi en Rattus spp. y $S$. hispidus, respectivamente, son las primeras reportadas en Venezuela.

Palabras clave: Leishmania guyanensis, Trypanosoma cruzi, Trypanosoma lewisi, reservorios de enfermedades, Venezuela.

Trypanosomatidae of public health importance occurring in wild and synanthropic animals of rural Venezuela

Introduction. Chagas disease and leishmaniasis are important public health problems due to their high frequency and broad distribution in Latin America. Understanding of the roles of reservoir animals is crucial for a global assessment of the epidemiology of these diseases.

Objective. To identify parasites classed as Trypanosomatidae as they occurred in sylvatic animals, and to establish rates of coinfection.

Materials and methods. Sylvatic animals were systematically captured in the rural area of El Carrizal, Merida State, Venezuela, betweenJuly, 1998 and February, 2000. The captures were 
made in Tomahawk type homemade traps, placed 15 nights per month throughout the study period. Blood was extracted from each captured and anesthetized animal by means of cardiac puncture. The search for trypanosomatids was undertaken by fresh blood examination, Giemsa stained blood smears and by means of blood-agar culture. Occasional xenodiagnoses were made to check diagnostic accuracy. The isolates obtained in culture media were identified by restriction fragment analysis and hybridization with specific probes.

Results. Three species of sylvatic animals $(n=215)$ were captured: Rattus spp. (135), Sigmodon hispidus (73) and Didelphis marsupialis (7). From them, three species of Trypanosomatidae were identified: Leishmania (Viannia) guyanensis, Trypanosoma cruzi and Trypanosoma lewisi. Trypanosoma. cruzi was identified in D. marsupialis (4/7), S. hispidus (1/73) and Rattus spp. (1/ 135), whereas L.(V.) guyanensis and T. lewisi were identified only in Rattus spp., 1/135 and 12/ 135 , respectively.

Conclusions. The coexistence of these genetically related hemoflagellates in sylvatic hosts was important for understanding the immunological interactions that may be established in reservoir animals, and the possible implications that this may have for the susceptible host. Finally, the identification of L.(V.) guyanensis in Rattus spp and T. cruzi in S. hispidus constituted the first reports of this relationship in Venezuela.

Keywords: Leishmania guyanensis, Trypanosoma cruzi, Trypanosoma lewisi, diseases reservoirs, Venezuela

Los parásitos de la familia Trypanosomatidae representan un verdadero problema de salud pública en Venezuela; se registran, por un lado, diferentes especies de Leishmania de los subgéneros Viannia y Leishmania, agentes causales de las diversas formas clínicas de leishmaniasis cutánea y de la forma visceral y, por el otro, Trypanosoma cruzi, agente causal de la enfermedad de Chagas. Estos dos grupos de protozoarios comparten las siguientes características: (i) en la naturaleza tienen una relación compleja con vectores invertebrados, donde cumplen parte de su ciclo, y que difieren para ambos grupos, así como con reservorios vertebrados, muchos de los cuales comparten, y un hospedero susceptible común, que es el ser humano; (ii) tienen una amplia distribución a lo largo de casi todo el continente americano y muchos de sus focos geográficos coinciden; (iii) la población en riesgo de contraer cualquiera de estas enfermedades en América es superior a los 100 millones de habitantes, los cuales fundamentalmente habitan en áreas rurales y suburbanas, dedicándose a las labores agrícolas

\footnotetext{
Correspondencia:

Hector de Lima, Instituto de Biomedicina, Universidad Central de Venezuela Apartado 4043, Caracas 1010A, Venezuela. Tele-fax: $58-212-8648624$

delimah@cantv.net
}

Recibido: 19/07/05; aceptado: 18/10/05 o pecuarias y, en la mayoría de los casos, con importantes restricciones económicas $(1,2)$.

En Venezuela las formas cutáneas de leishmaniasis tienen una amplia distribución a nivel nacional. Se han registrado casos en 23 de las 24 entidades federales; la única excepción es el estado Delta Amacuro. Para el decenio 19942003, a pesar del subregistro existente se reportaron 24.051 casos de leishmaniasis cutánea, con un promedio anual de 2.405 casos. Esto refleja una tasa anual promedio muy cercana a 10 por 100.000 habitantes (3).

Por otra parte, la transmisión de T. cruzi en Venezuela, aunque geográficamente algo más limitada que la de la leishmaniasis, abarca toda la región andina y de la costa, pero fundamentalmente se mantiene con índices importantes de transmisión en los estados Barinas, Lara y Portuguesa $(2,4,5)$. Hasta 1980, la enfermedad de Chagas se consideraba una de las cinco primeras causas de muerte a nivel nacional. En la medida en que los programas de control se han ido implementando ha descendido a las posiciones decimotercera y decimoctava, con un promedio anual de aproximadamente 720 muertes, $(4,5)$.

Una gran variedad de mamíferos han sido implicados como reservorios naturales de estas enfermedades, y muchos de ellos son comunes a ambas $(1,2)$. Además, algunos de estos 
reservorios son infectados por otros parásitos pertenecientes a la misma familia Trypanosomatidae, tales como Trypanosoma lewisi y Trypanosoma rangeli. El hecho de que todos estos parásitos mantengan una estrecha relación genética y compartan muchas características antigénicas nos plantea la posibilidad de que su interacción en ciertos focos, donde comparten determinados animales reservorios, implique una variación en su comportamiento con respecto a (i) la susceptibilidad (incrementándola o disminuyéndola) de ciertos reservorios a la infección por parásitos de la misma familia (Trypanosomatidae) o (ii) a su capacidad para infectar al hospedero susceptible.

Por ello, en el presente trabajo nos planteamos identificar las diversas especies de parásitos de la familia Trypanosomatidae en animales silvestres por medio de técnicas tradicionales morfológicas, biológicas y de biología molecular en un área endémica de leishmaniasis cutánea, en la cual no tenemos reportes de la existencia de la enfermedad de Chagas.

\section{Materiales y métodos}

\section{Área de estudio}

El Carrizal $\left(08^{\circ} 17^{\prime} 40^{\prime \prime N}-71^{\circ} 45^{\prime} 51^{\prime \prime O}\right)$ es una pequeña comunidad rural localizada en el municipio Tovar al suroeste del estado Mérida en Venezuela (figura 1), tradicionalmente endémica para leishmaniasis cutánea; se reportaron 15 casos durante la realización del estudio (3), mientras que no se detectaron casos de Chagas. Tiene una población estimada de cerca de $\mathbf{4 0 0}$ habitantes que viven dispersos en un área de unos 6 kilómetros cuadrados, dedicados en su mayoría a las labores agrícolas; su principal rubro es el café. El área en estudio es un bosque siempre verde que se ubica en la cordillera de los Andes entre 1.200 y 1.300 metros sobre el nivel del mar, con una temperatura promedio de $21^{\circ} \mathrm{C}$ y altamente intervenido por la presencia de múltiples sembradíos intercalados con las viviendas y áreas boscosas vírgenes. El estado Mérida, con una población estimada de 750.000 habitantes, se ubica en el suroeste del país, y en el periodo comprendido entre 1994 y 2003 reportó casi el $9,78 \%$ del total de casos de leishmaniasis cutánea a nivel nacional (3), lo que corresponde a una tasa de 33,27 por 100.000 habitantes.

\section{Captura de animales}

Los animales fueron capturados durante 19 meses entre julio de 1998 y febrero de 2000. Las trampas se colocaban quince días por mes en horas nocturnas adentro y alrededor de las viviendas, así como en las áreas de cultivos. Se utilizaron trampas de fabricación casera tipo Tomahawk. Como cebo se empleó cambur (plátano) y maíz. Los animales se mantuvieron vivos en cautiverio hasta el momento de ser estudiados.

\section{Métodos diagnósticos}

Los animales fueron examinados en la búsqueda de lesiones sospechosas, tales como úlceras, escoriaciones, nódulos y áreas de alopecia. Bajo anestesia se procedió a tomar una muestra de sangre por punción cardiaca con la cual se realizaban el examen en fresco, el extendido para coloración con Giemsa y el cultivo en medio agarsangre. Si alguno de estos exámenes resultaba positivo para Trypanosomatidae, el animal se sacrificaba en cámara de éter y se tomaban muestras de piel, hígado y bazo para el estudio histopatológico y el cultivo. Se realizó xenodiagnóstico en un número limitado de animales, empleando cinco ninfas de tercer estadio de Rhodnius prolixus. El contenido fecal de los triatominos se examinaba semanalmente durante un mes a partir de la primera semana posterior a su alimentación; de resultar negativos tres o cuatro exámenes, se descartaban los insectos.

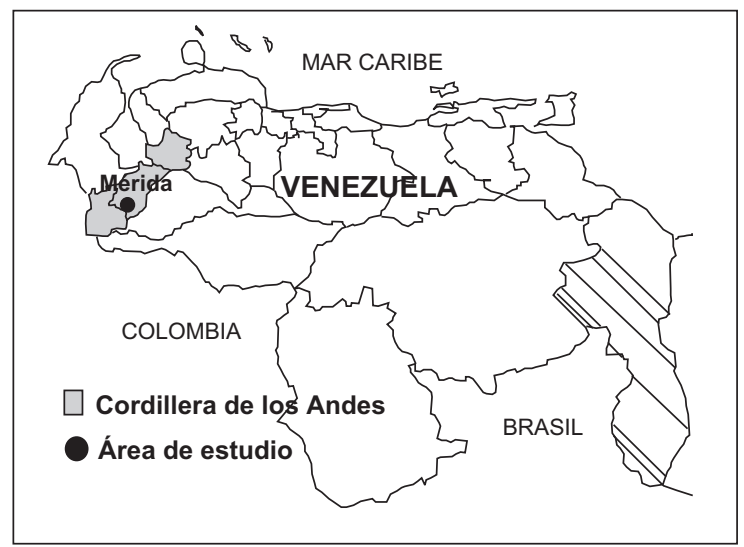

Figura 1. Área de estudio 


\section{Aislamiento y cultivo de parásitos}

Los parásitos se cultivaron en medio agar-sangre (Difco) con suplemento de $10 \%$ de sangre de conejo desfibrinada más 200 unidades de penicilina cristalina por mililitro. Los cultivos se examinaban al microscopio cada 7 días y los no contaminados se mantenían en observación durante un mes y luego se descartaban si permanecían negativos. Una vez que los cultivos se hacían positivos, se amplificaban en cajas de Petri que contenían el mismo medio. Los parásitos en fase estacionaria eran cosechados por centrifugación a 5.000 rpm durante 10 minutos a $4^{\circ} \mathrm{C}$ y, luego, lavados tres veces con tampón fosfato-salino estéril, $\mathrm{pH}$ 7,4.

\section{Aislamiento y digestión del ADNk}

El ADN del cinetoplasto se aisló tal como lo reportaron Rodríguez et al. (6). Para los análisis de esquizodemo se digirieron $5 \mu \mathrm{g}$ de ADNk con 5 unidades de la enzima Msp I (Gibco-BRL). Los productos digeridos se separaron por electroforesis en geles de poliacrilamida con un gradiente lineal $(4,5 \%$ a $10 \%)$ y se corrieron a 7 mAmp durante toda la noche. El gel se coloreó con nitrato de plata y, finalmente, fue fotografiado. La hibridación con sondas específicas se realizó tal como se describe en Barrios et al. (7). La prehibridación y la hibridación se realizaron durante toda la noche a $42^{\circ} \mathrm{C}$ en un tampón que contenía formamida al $50 \%$. Los filtros fueron revelados usando el Sistema Genios (Boehringer-Mannheim) según las instrucciones del fabricante.

\section{Aspectos éticos}

Los animales estudiados fueron tratados bajo las normas y principios de la guía internacional de principios para la investigación biomédica que involucra animales (8,9). Así mismo, se solicitó la correspondiente autorización del Comité de Bioética de nuestra institución, y se obtuvo la aprobación necesaria.

\section{Resultados}

Se capturaron y estudiaron 215 animales pertenecientes a tres especies (cuadro 1): Rattus spp. (135), S. hispidus (73) y D. marsupialis (7). En el examen físico, estos animales no revelaron lesiones de enfermedad alguna. Los resultados obtenidos por los cuatro diferentes métodos empleados para la identificación y diagnóstico de las diferentes especies de Trypanosomatidae se resumen en el cuadro 2. En D. marsupialis, considerando todos los métodos, se encontró un $57,14 \%(4 / 7)$ de los ejemplares estudiados infectado por flagelados que, basándonos en criterios morfológicos y en la positividad de 2/7 xenodiagnósticos, fueron clasificados como $T$. cruzi. Todos los casos con examen en fresco positivo en $D$. marsupialis se caracterizaron por una parasitemia extremadamente baja (1 parásito/ 50 campos de $40 \mathrm{X})$.

Cuadro 1. Trypanosomatidae identificados en animales silvestres capturados en el carrizal, estado Merida, Venezuela

\begin{tabular}{lrl}
\hline Nombre científico & $\mathbf{n}$ & $\begin{array}{c}\text { Trypanosomatidae } \\
\text { identificados }\end{array}$ \\
\hline $\begin{array}{l}\text { Roedores } \\
\text { Sigmodon hispidus }\end{array}$ & 73 & $\begin{array}{l}\text { Trypanosoma cruzi (1) } \\
\text { Rattus spp. } \\
\text { Trypanosoma cruzi (1) } \\
\text { Trypanosoma lewisi (12) }\end{array}$ \\
$\begin{array}{l}\text { Marsupiales } \\
\text { Didelphis } \\
\text { marsupialis }\end{array}$ & 735 & Trypanosoma cruzi (4) \\
Total & 215 & \\
\hline
\end{tabular}

Cuadro 2. Prevalencia de Trypanosomatidae en animales silvestres discriminados por método diagnóstico.

\begin{tabular}{|c|c|c|c|c|c|c|c|c|}
\hline \multirow[b]{2}{*}{ Nombre científico } & \multicolumn{2}{|c|}{ Exámen en fresco } & \multicolumn{2}{|c|}{ Frotis } & \multicolumn{2}{|c|}{ Cultivo } & \multicolumn{2}{|c|}{ Xenodiagnóstico } \\
\hline & Positivos & $\%$ & Positivos & $\%$ & Positivos & $\%$ & Positivos & $\%$ \\
\hline Sigmodon hispidus & $0 / 73$ & 0 & $0 / 73$ & 0 & $1 / 73$ & 1,37 & $0 / 10$ & 0 \\
\hline Rattus spp. & $12 / 135$ & $8,88^{a}$ & $12 / 135$ & $8,88^{a}$ & $13 / 135$ & $9,62^{b}$ & $0 / 15$ & 0 \\
\hline Didelphis marsupialis & $3 / 7$ & $42,85^{\circ}$ & $3 / 7$ & $42,85^{c}$ & $2 / 7$ & 28,57 & $2 / 7$ & 28,57 \\
\hline
\end{tabular}

a: todos identificados como T. lewisi; ${ }^{\text {b: }} 11$ cultivos sin identificar por crecimiento insuficiente; ${ }^{c}$ : todos identificados morfológicamente como T. cruzi. 
En Rattus spp., en el examen en fresco se observaron flagelados en 12/135 ejemplares (8,88\%); todos los casos fueron morfológicamente característicos de $T$. lewisi. Contrariamente a lo observado en D. marsupialis, los xenodiagnósticos realizados en Rattus resultaron todos negativos y las parasitemias observadas fueron bastante altas (5 a 10 parásitos/campo de 40X). En lo que respecta a $S$. hispidus, no se observaron parásitos en el examen en fresco ni por xenodiagnóstico.

De 16 cultivos obtenidos en las tres especies de animales estudiadas, sólo tres ( $S$. hispidus, 1; Rattus spp., 2) crecieron y se adaptaron adecuadamente en el medio, lo que permitió su identificación taxonómica por técnicas de biología molecular. Todos los cultivos se obtuvieron a partir de muestras de sangre, ninguno de los órganos cultivados (bazo, hígado, piel) dio cultivo positivo. La figura 2 muestra los patrones de restricción de las tres cepas aisladas después de la digestión con la endonucleasa Msp I. La muestra 1

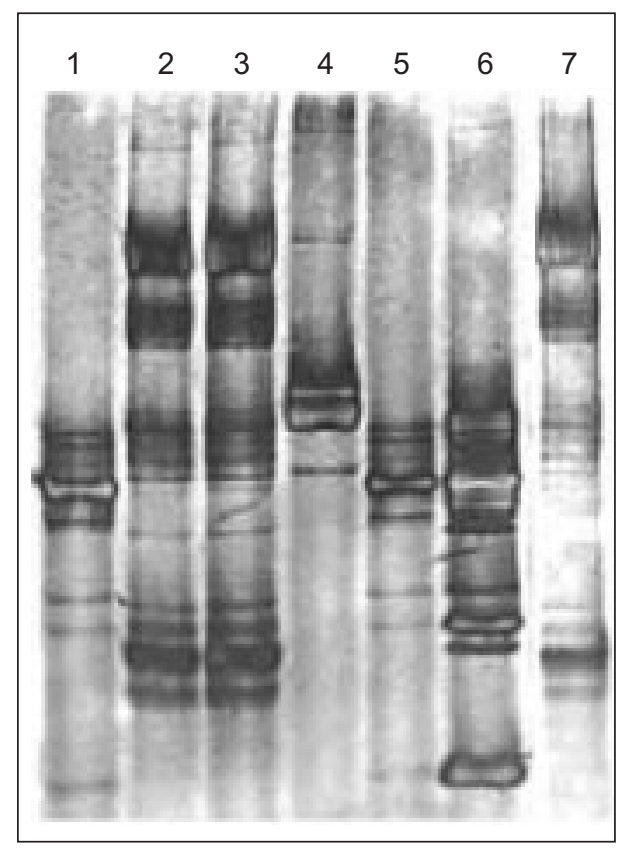

Figura 2. Patrones de restricción después de la digestión con la enzima Msp I. Orden de las muestras: 1. aislamiento de Rattus spp.; 2. aislamiento de $S$. hispidus; 3. aislamiento de Rattus spp.; 4. L(V) braziliensis; 5. L(V) guyanensis; 6. $L(L)$ amazonensis; 7. T. cruzi. (aislamiento de Rattus) presentó un patrón de restricción similar al de L. (V.) guyanensis (M4147) (línea 5). Este resultado fue confirmado después de la hibridación con sondas para $L$. (V.) braziliensis (figura $3 \mathrm{~A}$ ), L. (V.) guyanensis (figura 3B) y L. (L.) amazonensis (figura 3C).

Las muestras 2 (aislamiento de Sigmodon) y 3 (aislamiento de Rattus) tuvieron un patrón de restricción similar a $T$. cruzi (línea 7), hecho confirmado por la hibridación con sondas para $L$. (V.) braziliensis, L. (V.) guyanensis, L. (L.) amazonensis y $T$. cruzi (resultados no mostrados).

\section{Discusión}

El estudio de los posibles reservorios silvestres y sinantrópicos de hemoflagelados zoonóticos es de gran relevancia a nivel mundial y en especial en Suramérica, en donde dos de sus representantes, Leishmania spp. y Trypanosoma cruzi, se han descrito en muchas especies de vertebrados con infección natural por alguno de estos parásitos y

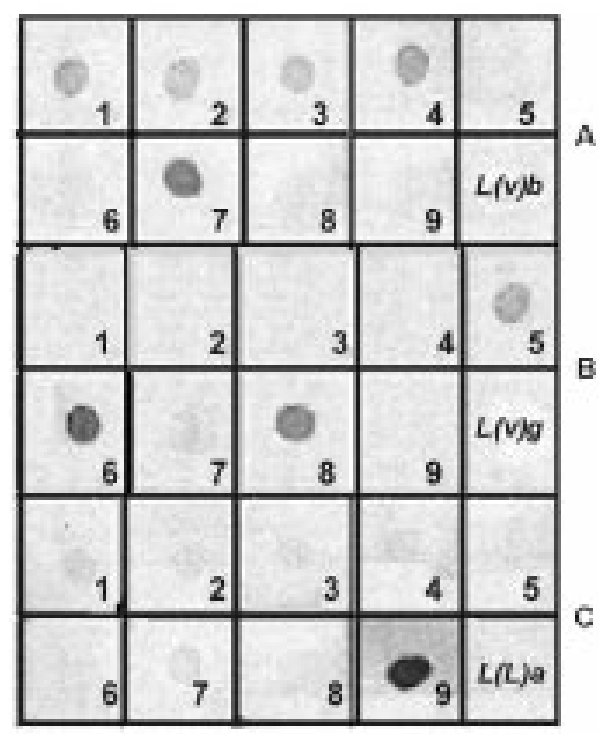

Figura 3. Hibridación con sondas especie específicas. A: con L.(V.) braziliensis (M2903 kADN). B: con L.(V.) guyanensis (M4147 kADN). C: con L.(L). amazonensis (PH8 kADN). Orden de las muestras: 1 a 5 pacientes. 6. Rattus ssp.7. L.(V.) braziliensis. 8. L.(V.) guyanensis. 9. L.(L.) amazonensis. 
en algunos casos por ambos $(1,2)$. Sin embargo, estos estudios no han evaluado la importancia epidemiológica de la coexistencia de diversas especies de hemoflagelados, incluidos algunos sin aparente importancia médica, en determinados animales vertebrados en un área geográfica delimitada. La mayoría de los estudios se han limitado a evaluar la presencia de sólo uno de ellos, bien sea Leishmania spp., T. cruzi o $T$. lewisi, sin pensar en la posibilidad de que la existencia previa de alguno de ellos pudiera significar cambios inmunológicos importantes en el vertebrado que determinen una mayor o menor susceptibilidad del mismo a otros parásitos, incluidas otras especies de hemoflagelados. Algunos estudios previos $(10,11)$ sugieren que la infección de ratas por $T$. lewisi genera un estado de inmunosupresión que incrementa su susceptibilidad a posteriores infecciones por Salmonella typhimurium y Toxoplasma gondii.

En Venezuela se han realizado pocos estudios para la identificación de reservorios silvestres de parásitos hemoflagelados; la mayoría de los trabajos se han dedicado al estudio de animales domésticos (12).

En el caso de Leishmania, los primeros animales silvestres descritos con infección natural fueron de la zona de Socopo, estado Barinas, donde se encontró en 1/29 Zygodontomys microtinus con amastigotes en una lesión en la cola, y posteriormente, en 1/80 Proechymys guyanensis se demostró la presencia de parásitos en una lesión ulcerada del animal (12). En ninguno de estos dos casos se identificó la especie de parásito involucrada. En $D$. marsupialis (2/40) se encontraron parásitos identificados como $L$. (V.) guyanensis en la ciudad de Trujillo (13). En un estudio realizado en el estado Lara (14), de 519 animales estudiados, pertenecientes a 10 especies diferentes, se confirmó la infección natural de $S$. hispidus (1/391) con L. (V.) braziliensis y de Rattus spp. (2/68) con $L$. (L.) mexicana y $(3 / 68)$ con $L$. ( $V$.) braziliensis.

En el presente estudio, de 215 animales estudiados, pertenecientes a tres especies, se logró demostrar la infección natural con $L$. (V.) guyanensis en Rattus spp. (1/135). A pesar de tratarse de una tasa de infección baja $(0,74 \%)$, consideramos que esta especie debe estar actuando como reservorio en el área de estudio con base en los siguientes criterios: (i) a partir de cultivos aislados en pacientes de la misma zona se logró identificar los parásitos pertenecientes a la misma especie de Leishmania (resultado no mostrado); (ii) las dificultades técnicas en el aislamiento y adaptación de parásitos en medios de cultivo, frecuentemente contribuyen a una subestimación de la tasa de infección; (iii) Rattus spp. ha sido implicado como reservorio de las diferentes especies de Leishmania a nivel mundial (en el Viejo Mundo se le implica más frecuentemente como reservorio de Leishmania causantes de la forma visceral $(15,16)$, mientras que en Suramérica se le asocia con mayor frecuencia a parásitos causantes de la forma cutánea $(14,17-20)$ y con menor frecuencia a parásitos del grupo donovani $(21,22)$; (iv) existen evidencias experimentales de que Rattus spp. actúa como un buen reservorio para parásitos del genero Leishmania; estos parásitos inyectados subcutáneamente en Rattus spp. producen una infección crónica sin la aparición de lesiones cutáneas, y conservan la capacidad de infectar al vector $(23,24)$. La ausencia de lesiones es compatible con lo observado por nosotros en el presente trabajo y en trabajos anteriores (14); (v). en Venezuela, Rattus es una especie bastante común en zonas rurales, suburbanas y urbanas (25), que habita en estrecha relación con el ser humano. La casi totalidad de las capturas son intradomiciliarias o provienen del peridomicilio inmediato. En este trabajo se reporta por primera vez la presencia de $L$. ( $V$.) guyanensis en Rattus.

El segundo hemoflagelado observado en el área en estudio fue $T$. cruzi, identificado en las tres especies de animales capturadas: $S$. hispidus (1/73), Rattus spp. (1/135) y D. marsupialis (4/7). Todos los Didelphi fueron capturados en el peridomicilio inmediato y $57,14 \%$ de ellos resultó positivo para T. cruzi. A pesar de esta alta tasa de infección, no hubo conocimiento sobre casos agudos ni crónicos de enfermedad de Chagas durante el periodo en que se desarrolló el proyecto (19 meses). Se requeriría la realización de nuevos estudios, sobre todo en lo referente a vectores, y 
un despistaje serológico en la población general para evaluar la situación real del foco y determinar si hay transmisión actual, si la hubo en el pasado o si nunca la ha habido.

En Venezuela, $T$. cruzi se ha encontrado infectando de forma natural 31 especies de vertebrados silvestres pertenecientes a 6 órdenes (2). El más frecuentemente encontrado es $D$. marsupialis (26-28), al igual que ocurre en el resto de Suramérica $(21,29-32)$. Por sus hábitos de vida representa un importante reservorio tanto del ciclo peridomiciliario como del selvático. $D$. marsupialis infectado con $T$. cruzi $(6 / 24,25 \%)$ ha sido reportado en áreas urbanas de Caracas (28). Se encontró Rattus spp. infectado en $0,74 \%$ de los animales examinados (1/135). La infección de Rattus por $T$. cruzi ya había sido reportada en Venezuela tanto en áreas urbanas (33) como rurales.

S. hispidus se encontró infectado por $T$. cruzi en $1,36 \%(1 / 73) y$, aunque su infección natural se ha reportado en Colombia y EI Salvador (2), éste es el primer reporte para Venezuela. Su importancia como reservorio del ciclo selvático está por demostrarse.

Finalmente, $T$. lewisi se encontró en $8,88 \%$ de Rattus spp. examinados (12/135). Aunque no tiene importancia aparente desde el punto de vista médico, pensamos que podría ser importante desde el punto de vista inmunológico. Estudios experimentales previos confirman que la infección por $T$. lewisi en Rattus genera un estado de inmunosupresión que intensifica infecciones por otros parásitos, tales como Salmonella typhimurium y Toxoplasma gondii $(10,11)$. En el presente estudio se encontró que de 135 animales estudiados, 12 estaban infectados con $T$. lewisi y dos de ellos estaban simultáneamente infectados con $T$. cruzi uno y con L.(V.) guyanensis el otro, mientras que los restantes 123 animales no presentaban ningún tipo de infección. Durante la realización de este estudio, se evaluó simultáneamente otro foco de leishmaniasis cutánea en el mismo estado Mérida, donde se capturaron 189 Rattus spp.; ninguno reportó infección con $T$. lewisi y en ellos tampoco pudo demostrarse infección por otros hemoflagelados, a pesar de que se siguieron los mismos procedimientos. En estudios anteriores (34), en los que se evaluó un foco activo de leishmaniasis cutánea e inactivo para enfermedad de Chagas, se procesaron 40 Rattus spp., y se obtuvo coinfección entre T. lewisi, Leishmania spp. y $T$. cruzi. Estudios realizados en Colombia (21) demuestran también la infección simultánea de D. marsupialis por T. cruzi y L. chagasi.

Los resultados obtenidos deben considerarse a la hora de evaluar los reservorios de nuevos focos tanto de leishmaniasis como de Chagas, ya que la infección con $T$. lewisi podría sensibilizar a los animales silvestres para convertirlos en portadores de otros flagelados de importancia médica. También se requiere la realización de experimentos controlados en el laboratorio que corroboren nuestra teoría.

\section{Agradecimientos}

A la comunidad de El Carrizal por todo el apoyo suministrado durante la realización del trabajo de campo.

\section{Conflicto de intereses}

Los autores declaran no tener conflicto de intereses en este trabajo.

\section{Financiación}

Banco Mundial / Gobierno de Venezuela, proyecto Ven/96/002/021-024.

\section{Referencias}

1. World Health Organization. Las leishmaniasis, informe de un comité de expertos de la OMS. Serie de informes técnicos 701. Geneve: WHO; 1984. p.23-74

2. World Health Organization. Second report of the WHO Expert Committee, Control of Chagas' disease. Technical report series 905. Geneve:WHO; 2002. p.5563.

3. Ministerio de Salud y Desarrollo Social, Venezuela. Servicio Autónomo Instituto de Biomedicina. Unidad de Informática. Informe anual de leishmaniasis cutánea en Venezuela. Caracas: Instituto de Biomedicina;19942003.

4. Ministerio de Salud y Desarrollo Social. 1980-2000. Anuario de epidemiología y estadística vital. Caracas: MSDS; 2001.

5. Aché A, Matos AJ. Interrupting Chagas disease transmission in Venezuela. Rev Inst Med Trop Sao Paulo 2001;43:37-43. 
6. Rodríguez N, Guzmán B, Rodas A, Takiff H, Bloom B, Convit J. Diagnosis of cutaneous leishmaniasis and species discrimination of parasite by PCR and hybridization. J Clin Microbiol 1994;32:2246-52.

7. Barrios MA, Rodríguez N, Feliciangeli DM, Ulrich M, Telles S, Pinardi ME et al. Coexistence of two species of Leishmania in the digestive tract of the vector Lutzomyia ovallesi. Am J Trop Med Hyg 1994;51:66975.

8. Bankowski Z, Howard-Jones N. Biomedical research involving animals: Proposed international guiding principles. Geneva: WHO; 1985.

9. Bankowski Z, Howard-Jones N. International guiding principles for biomedical research involving animals. Geneva: WHO; 1986.

10. Nielsen K, Sheppard J, Holems W, Tizard I. Increased susceptibility of Trypanosoma lewisi infected, or decomplemented rats to Salmonella typhimurium. Experientia 1978;34:118-9.

11. Guerrero OM, Chinchilla M, Abrahams E. Increasing of Toxoplasma gondii (Coccidia: Sarcocystidae) infections by Trypanosoma lewisi (Kinetoplastida, trypanosomatidae) in white rats. Rev Biol Trop 1997;45:877-82.

12. Bonfante-Garrido R, Barroeta S. Leishmanias y leishmaniasis en América con especial referencia a Venezuela. Barquisimeto: Tipografía y Litografía Horizonte C. A.; 2002. p.39-73

13. Scorza JV, Rezzano S, Márquez JC. Didelphis marsupialis: reservorio primario de Leishmania spp. en la ciudad de Trujillo, Venezuela. Rev Cub Med Trop 1984;36:194-200.

14. De Lima H, De Guglielmo Z, Rodríguez A, Convit J, Rodríguez N. Cotton rats (Sigmodon hispidus) and black rats (Rattus rattus) as possible reservoirs of Leishmania spp in Lara state, Venezuela. Mem Inst Oswaldo Cruz 2002;97:169-74.

15. Gradoni L, Pozio E, Gramiccia M, Maroli M, Bettini S. Leishmaniasis in Tuscany (Italy): VII. Studies on the role of the black rat, Rattus rattus, in the epidemiology of visceral leishmaniasis. Trans R Soc Trop Med Hyg 1983;77:427-31.

16. Ibrahim EA, al-Zahrani MA, al-Tuwaigri AS, alShammary FJ, Evans DA. Leishmania infecting man and wild animals in Saudi Arabia. 9. The black rat (Rattus rattus) a probable reservoir of visceral leishmaniasis in Gizan province, south-west Saudi Arabia. Trans R Soc Trop Med Hyg 1992;86:513-4.

17. Johnson RN, Young DG, Butler JF, Bogaert-Diaz H. Possible determination of the vector and reservoir of leishmaniasis in the Dominican Republic. Am J Trop Med Hyg 1992;46:282-7.

18. Vasconcelos IA, Vasconcelos AW, Fe Filho NM, Queiroz RG, Santana EW, Bozza M et al. The identity of Leishmania isolated from sand flies and vertebrate hosts in a major focus of cutaneous leishmaniasis in Baturite, northeastern Brazil. Am J Trop Med Hyg 1994;50:158-64.

19. Alexander B, Lozano C, Barker DC, McCann SH, Adler GH. Detection of Leishmania (Viannia) braziliensis complex in wild mammals from Colombian coffee plantations by PCR and DNA hybridization. Acta Trop 1998;69:41-50.

20. Brandao-Filho SP, Brito ME, Carvalho FG, Ishikawa EA, Cupolillo E, Floeter-Winter $L$ et al. Wild and synanthropic hosts of Leishmania (Viannia) braziliensis in the endemic cutaneous leishmaniasis locality of Amaraji, Pernambuco State, Brazil. Trans R Soc Trop Med Hyg 2003;97:291-6.

21. Travi BL, Jaramillo C, Montoya J, Segura I, Zea A, Goncalves A et al. Didelphis marsupialis, an important reservoir of Trypanosoma (Schizotrypanum) cruzi and Leishmania (Leishmania) chagasi in Colombia. Am J Trop Med Hyg 1994;50:557-65.

22. Zulueta AM, Villarroel E, Rodriguez N, Feliciangeli MD, Mazzarri M, Reyes $\mathrm{O}$ et al. Epidemiologic aspects of American visceral leishmaniasis in an endemic focus in Eastern Venezuela. Am J Trop Med Hyg 1999;61:94550.

23. Pozio E, Maroli M, Gradoni L, Gramiccia M. Laboratory transmission of Leishmania infantum to Rattus rattus by the bite of experimentally infected Phlebotomus perniciosus. Trans R Soc Trop Med Hyg 1985;79:524-6.

24. Svobodova M, Votypka J, Nicolas L, Volf P. Leishmania tropica in the black rat (Rattus rattus): persistence and transmission from asymptomatic host to sand fly vector Phlebotomus sergenti. Microbes Infect 2003;5:361-4.

25. Camero CL, Gómez WE, Cáceres JL. Infestación por roedores en inmuebles de Turmero, estado Aragua, Venezuela, 2001. Boletín de Malariología y Salud Ambiental 2004;XLIV:29-33.

26. Telfort Jr SR, Tonn R. Dinámica de Trypanosoma cruzi en poblaciones de un reservorio primario, Didelphis marsupialis, en los llanos altos de Venezuela. Bol Oficina Sanit Panam 1982;93:341-64.

27. Gil de Soto ST. Didelphis marsupialis, Linnaeus 1758 , su importancia como reservorio de Trypanosoma cruzi en la región de Zipanare (estado Zulia, Venezuela). Kasmera 1971;4:5-44.

28. Herrera L, Urdaneta-Morales S. Didelphis marsupialis: a primary reservoir of Trypanosoma cruzi in urban area of Caracas, Venezuela. Ann Trop Med Parasitol 1992;86:607-12.

29. Grisard EC, Carvalho-Pinto CJ, Scholz AF, Toma HK, Schlemper BR Jr, Steindel M. Trypanosoma cruzi infection in Didelphis marsupialis in Santa Catarina 
and Arvoredo Islands, southern Brazil. Mem Inst Oswaldo Cruz 2000;95:795-800.

30. Ramirez LE, Lages-Silva E, Alvarenga-Franco $F$, Matos A, Vargas $\mathrm{N}$, Fernandes $\mathrm{O}$ et al. High prevalence of Trypanosoma rangeli and Trypanosoma cruzi in opossums and triatomids in a formerly-endemic area of Chagas disease in Southeast Brazil. Acta Trop 2002;84:189-98.

31. Wisnivesky-Colli C, Schweigmann NJ, Alberti A, Pietrokovsky SM, Conti O, Montoya S et al. Sylvatic American trypanosomiasis in Argentina. Trypanosoma cruzi infection in mammals from the Chaco forest in Santiago del Estero. Trans R Soc Trop Med Hyg 1992;86:38-41.
32. Raccurt CP. Trypanosoma cruzi in French Guinea: review of accumulated data since 1940. Med Trop 1996;56:79-87.

33. Herrera L, Urdaneta-Morales S. Synanthropic rodent reservoirs of Trypanosoma (Schizotrypanum) cruzi in the valley of Caracas, Venezuela. Rev Med Trop Sao Paulo 1997;39: 279-82.

34. De Lima H, Rodriguez N, Borges R, Convit J. Trypanosoma (Herpetosoma) lewisi in Rattus spp from La Matica, Lara State, Venezuela and the possible relationship with zoonotic diseases. Boletín de Malariología y Salud Ambiental 2003;XLIII:37-43.

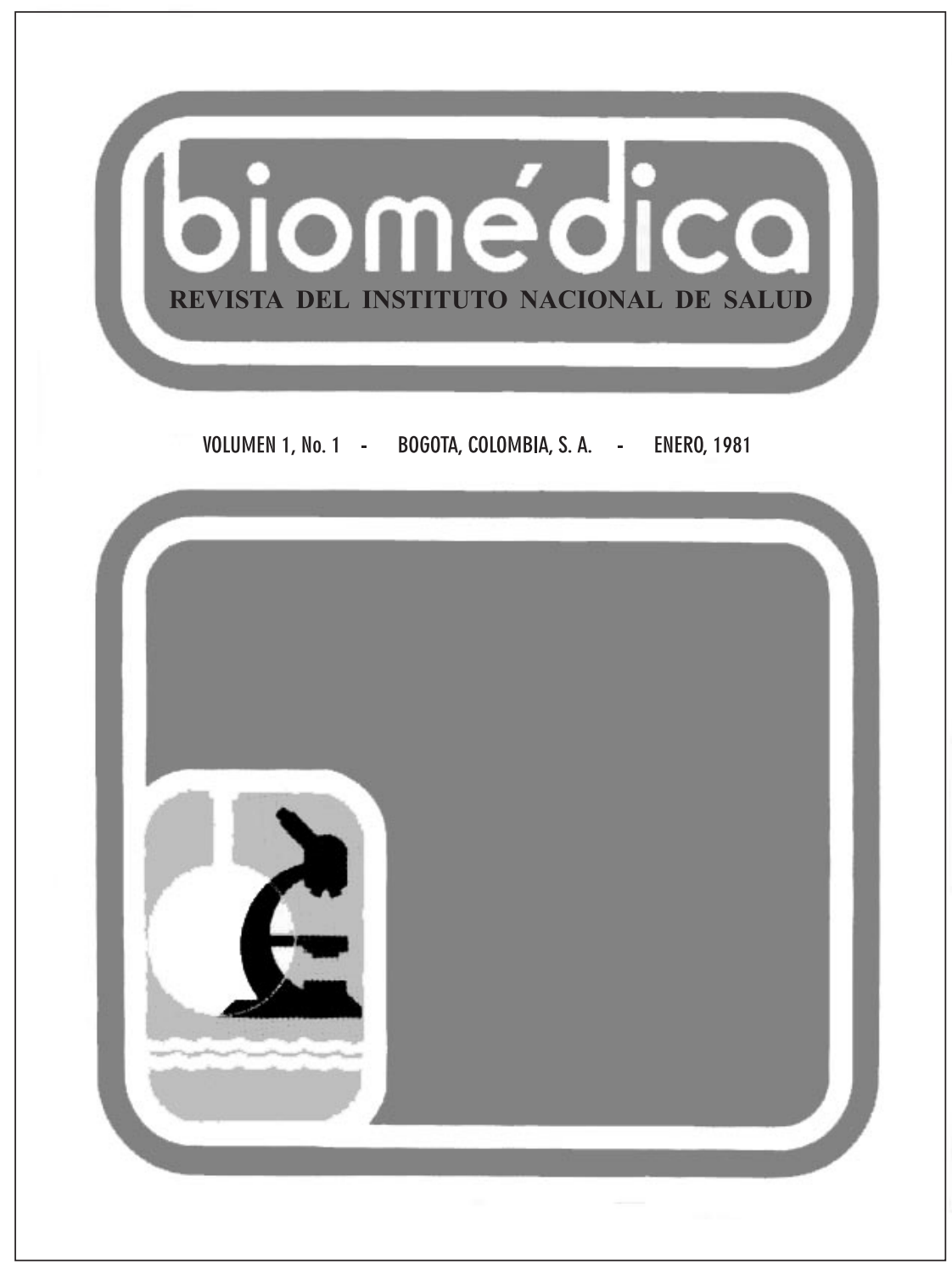

